\title{
AIAA 2001-0529
}

Unsteady Flow Investigations in an Axial Turbine Using the Massively Parallel Flow Solver TFLO

Jixian Yao, Roger L. Davis, Juan J. Alonso, and Antony Jameson

Stanford University, Stanford, CA 94305 and United Technologies Research Center, E. Hartford, CT 06108

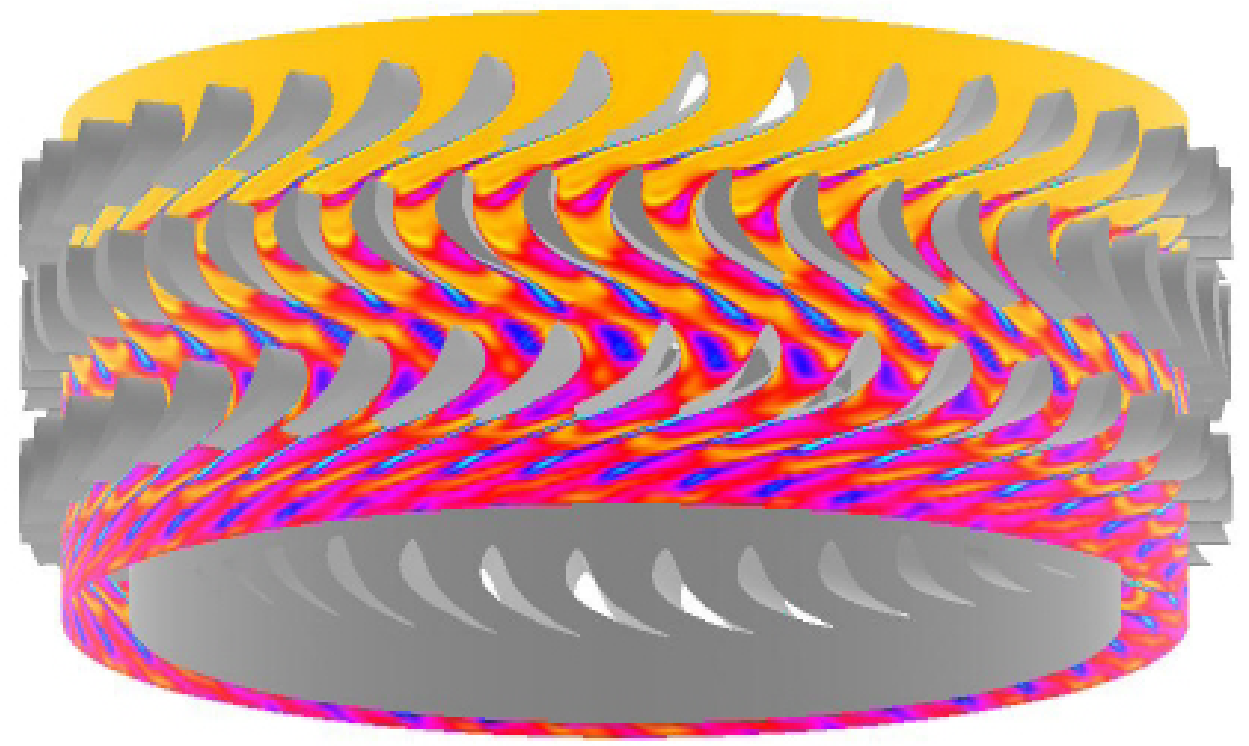

\section{9th AIAA Aerospace Sciences Meeting and Exhibit January 8-11, 2001/Reno, NV}


AIAA 2001-0529

\title{
Unsteady Flow Investigations in an Axial Turbine Using the Massively Parallel Flow Solver TFLO
}

\author{
Jixian Yao, Roger L. Davis ${ }^{\dagger}$ Juan J. Alonso; and Antony Jameson ${ }^{\ddagger}$ \\ Stanford University, Stanford, CA 94305 and \\ United Technologies Research Center, E. Hartford, CT 06108
}

\begin{abstract}
The results from two numerical simulations of the unsteady flow in a 1-1/2 stage axialflow turbine are presented and compared with experimental data to show both the effect of blade count on the solution accuracy and the time-averaged and unsteady flow physics present. The TFLO three-dimensional, multi-block, massively parallel turbomachinery flow solution procedure is used to simulate the flow through the Aachen 36-vane/41blade/36-vane 1-1/2 stage turbine rig. Comparisons of the time-averaged and unsteady flow solutions of 1-vane/1-blade/1-vane and 6-vane/7-blade/6-vane configurations with the available experimental data are used to show the importance of matching actual blade counts in unsteady flow simulations as closely as possible. In addition, these comparisons are used to quantify the predicted aerodynamic performance differences and highlight the different unsteady flow physics in the two simulations.
\end{abstract}

\section{Introduction}

Accurate prediction of the flow physics in turbomachinery has been the subject of intensive research for many years. Unsteady flow phenomena in turbomachinery has especially been of great interest recently in an effort to improve aerodynamic efficiency, stability, and operability. It is now possible with today's high performance parallel computer systems to simulate the flow field in turbomachinery with fewer assumptions and simplifications. Together with experimental investigations, numerical simulations can lead to a deeper understanding of the unsteady and timeaveraged flows in turbomachinery and to subsequent improvements in turbomachinery design.

In an effort to stimulate the application of massively parallel computer systems and the development of numerical simulation tools that can take advantage of these systems, the Department of Energy (DoE) launched the Accelerated Strategic Computing Initiative (ASCI). The three-dimensional, multiblock, parallel flow solver, $\mathrm{TFLO}^{1}$ has been developed under this initiative in an effort to step up to large-scale parallel steady and unsteady flow multi-stage turbomachinery simulations. The unsteady Reynolds-averaged NavierStokes equations are solved in the TFLO procedure

\footnotetext{
* Research Associate, Member AIAA

${ }^{\dagger}$ Senior Consulting Engineer of United Technologies Research Center, Member AIAA

$\ddagger$ Assistant Professor, Member AIAA

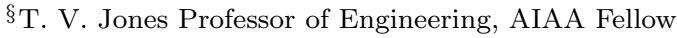

Copyright (C) 2001 by the authors. Published by the American Institute of Aeronautics and Astronautics, Inc. with permission.
}

using a cell-centered discretization on arbitrary multiblock meshes. The solution algorithm is based on an efficient implicit, dual time-step procedure ${ }^{2}$ in which an explicit Runge-Kutta integration scheme coupled with multigrid, implicit residual smoothing, and local time-stepping convergence acceleration techniques is used in an inner iteration for each time-step. Wilcox's ${ }^{3}$ $k$ - $\omega$ two-equation turbulence model is used to predict the turbulence viscosity in the field. The solver is parallelized using domain decomposition, an SPMD (Single Program Multiple Data) strategy, and the Message Passing Interface (MPI) standard. Unsteady multiblade row simulations are performed with the TFLO code using reduced integer blade counts that match or closely approximate those of the actual configuration.

In the current investigation, the TFLO solution procedure has been used to investigate the unsteady flow through the Aachen 1-1/2 stage axial flow turbine documented as an ERCOFTAC (European Research Community On Flow, Turbulence And Combustion) benchmark test case. The experimental measurements were performed by Walraevens and Gallus. ${ }^{4}$ Previous steady flow calculations have been reported by Emunds et. al. ${ }^{5}$ and unsteady flow calculations have been reported by Walraevens et. $\mathrm{al}^{6}$ and by Volmar et. al. ${ }^{7}$ Those investigations were mainly focused on the secondary flow features and used a single passage per blade row with either phase-lagged boundary conditions $^{8}$ or time-inclining ${ }^{9}$ to account for different airfoil counts in each blade row.

Unfortunately, even with large massively parallel 
computer systems, it is not always possible to simulate all of the blade passages included in the entire $360^{\circ}$ of the turbomachine wheel. When the blade counts across the machine can be divided by some common factor, simulations can be executed with a reduced circumferential period that is common to all the blade rows in the configuration. This reduced circumferential period is usually kept as small as possible in order to minimize the required computational resources. Frequently, however, it is not possible to come to some common circumferential period across the blade rows due to blade counts that are not divisible by a common factor. In this case, the blade counts are modified as little as possible to allow a reduction in the solution domain. Since the airfoil loading of a given blade row is a function of the number of blades, different scaling strategies can be used on the airfoil to reduce the aerodynamic effect of changing the number of blades.

Unsteady multi-blade row simulations are performed with the TFLO code using a reduced integer blade count for each blade row that closely provides for a common global circumferential pitch in all blade rows. To achieve an exact common global pitch, however, one or more of the blade rows must be scaled by the ratio of the actual blade count to the simulation blade count. In the current investigation, airfoils to be modified due to a change in the blade count are scaled in the axial and circumferential directions in order to hold blade pitch/chord ratio and loading levels as consistent as possible with the original blade row. The advantage of the reduced integer blade count strategy is that storage requirements are kept to a minimum, inverse transformations are not required to post-process the results, and low-frequencies (below the first bladepassing frequency) are admitted which can especially be important at off-design conditions.

In order to quantify the effect of using this type of blade count scaling on the accuracy of flow prediction, two different unsteady flow simulations have been performed. The simpler of the two simulations uses only one blade passage per blade row (1-1-1), and the more exact first-time simulation solves the flow in 6 passages in the first stator, 7 passages in the rotor, and 6 passages in the second stator (6-7$6)$. Comparisons between the time-averaged solutions and experimental data show only minor differences in the pressure loading levels of each blade. In addition, the unsteady pressure envelopes of each blade row compare favorably between the two simulations. However, Fourier decompositions of the unsteady pressure fields show significant differences in the unsteady pressure amplitudes between the 1-1-1 and the 6-7-6 solutions. Similar findings have been found by Clark, et. al. ${ }^{10}$ in an investigation of a $1-1 / 2$ stage transonic turbine. As mentioned by Clark, et. al., the differences in harmonic amplitudes predicted by the simulations can be a significant factor in the accurate prediction of resonance stress levels on the blades. In addition, the current investigation has determined that the predicted relative total pressure losses can be significantly different due to the scaling strategy. Investigation of the unsteady relative total pressure loss field shows differences in the loss migration and mixing between the two simulations. These findings lead to the conclusion that the scaling factor used in these types of multiblade row unsteady flow should be kept to a minimum in order to accurately predict time-averaged total pressure losses and peak unsteady pressure loadings.

\section{The Aachen 1.5-Stage Axial Turbine}

The Aachen turbine configuration is a subsonic axial flow rig consisting of three blade rows: the first vane, the blade, and the second vane. The geometry of the second vane is exactly the same as that of the first vane. A schematic of the configuration is presented in Figure 1. The exact blade counts for this case are 36, 41, and 36 respectively. The geometry and experimental data package were provided by ERCOFTAC (European Research Community On Flow, Turbulence And Combustion). There are two operating points given in the ERCOFTAC package, one with a lower mass flow rate $(7 \mathrm{~kg} / \mathrm{s})$ and another with a higher mass flow rate $(8 \mathrm{~kg} / \mathrm{s})$. The lower mass flow case is calculated in this paper.

A steady flow calculation was performed using a simple mixing plane between adjacent blade rows. This calculation served as a baseline for comparison with the unsteady flow solutions and the experimental data as well as the initial conditions for the unsteady flow simulations.

Two unsteady flow calculations have been performed with different passage counts per blade row as previously mentioned: the 1-1-1 and the 6-7-6 vane/blade/vane simulations. The unsteady flow solutions were time-averaged over each global period to compare with the steady flow solution and experimental data. The unsteady flow solutions were also Fourier decomposed in an effort to understand the various unsteady pressure modes in the flow fields.

\section{Configuration 1-1-1}

This configuration consists of one blade passage per blade row, the geometry of the two vanes is kept unchanged, while the geometry of the blade is scaled to form 36 blade passages. Comparing to the original blade count of 41 , the rotor blade is actually enlarged, but the solidity $(t / C)$ is kept unchanged. Also, the axial distances between two adjacent blade rows are not changed. The scaling factor of the blade is 1.1389 .

The mesh sizes used in this calculation can be summarized as follows: 1st vane: $137 \times 65 \times 81$, rotor: $113 \times 65 \times 81$ for the main passage and $89 \times 17 \times 17$ for the tip gap, stator: $153 \times 65 \times 81$. This resulted in 

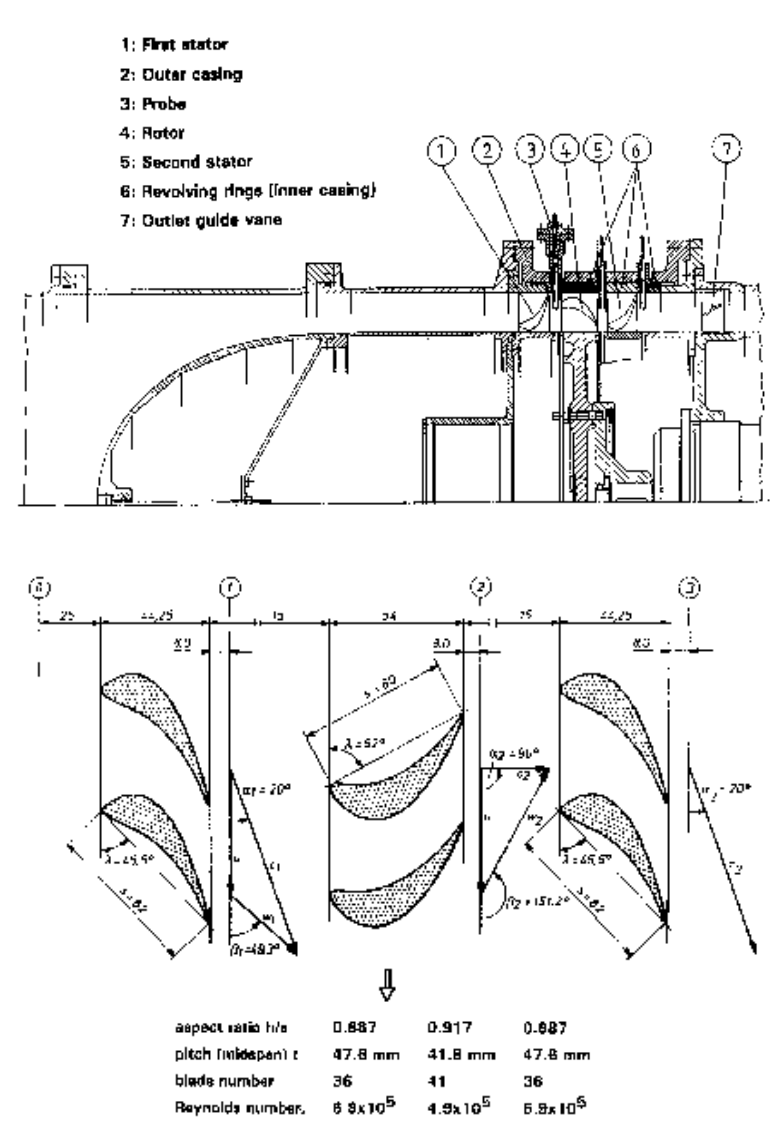

Fig. 1 Schematic of the Aachen 1.5-stage turbine

a total of $2,147,516$ points.

\section{Configuration 6-7-6}

Compared with the above configuration, this configuration consists of 6 passages for the 1st vane, 7 passages for the blade, and 6 passages for the second vane. The geometry of the vanes are kept unchanged like in the 1-1-1 configuration, while the rotor blade is slightly shrunk to form a blade count of 42 . This is the closest common blade count for this turbine rig, and the blade is only shrunk by a factor of 0.9762 . Similarly to the 1-1-1 configuration, the solidity of the blade and the axial distances between two adjacent blade rows are not changed. Figure 2 shows the computational grid for this configuration. The grid sizes for each blade passage are the same as those of the 1-1-1 configuration. This leads to a total of $13,505,762$ points.

\section{Estimate of Computational Complexity}

In order to accurately resolve the waveforms corresponding to the stator-rotor-stator interaction, a total of 100 time steps were used to rotate one blade past one vane passage. This resulted in the use of 700 time steps for one global period corresponding to $\frac{1}{6}$ of the turbomachine circumference. For each time step, 30 multigrid cycles are performed to ensure the conver-

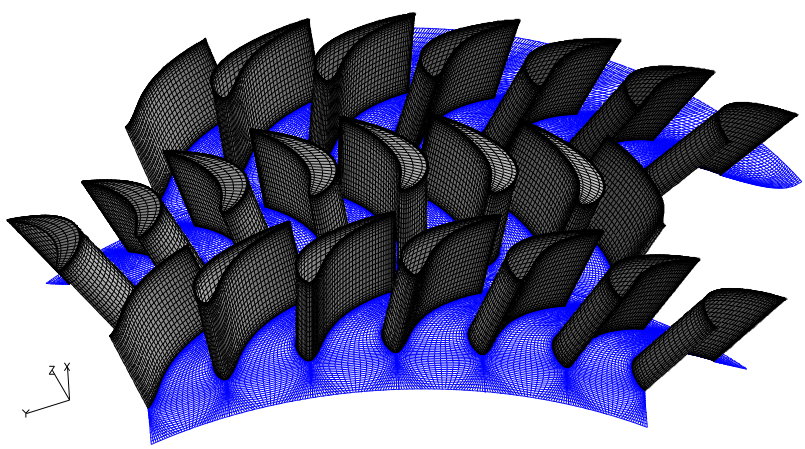

Fig. 2 Computational grid for configuration 6-7-6, every other grid point shown

gence of the inner iteration. The 6-7-6 simulation was executed on 187 processors of the DoE Blue Pacific IBM SP2 at Lawrence Livermore Laboratory. Each time step (with 30 inner iterations) required approximately 27.3 minutes including the computational and restart file input/output time. The 1-1-1 simulation was executed on 12 processors of an SGI Origin 2000. Each time step required approximately 46.6 minutes. The parallel computational efficiency of the TFLO code for up to 1024 processors is documented in Ref. ${ }^{11}$ Eleven global cycles (or 11 vane passings by the blade) were executed in order to reach a time periodic solution for the 1-1-1 configuration. Four global cycles (or 24 vane passings by the blade or 28 blade passings by a vane) were executed for the 6-7-6 configuration.

\section{Time-Averaged Performance Parameters}

The details of the time-averaged overall performance characteristics are discussed in this section. These time-averaged results, together with the results of a steady flow calculation, are compared to the experimental data available at $8.8 \mathrm{~mm}$ downstream of the trailing edges of the three blade rows.

\section{Vane-1 Exit}

A comparison between the predicted and experimental circumferentially averaged absolute total pressure at the exit of vane-1 $(8.8 \mathrm{~mm}$ downstream of the trailing edge) is shown in Figure 3. The inlet experimental total pressure profile which was used in all of the simulations as an upstream boundary condition is also included as a reference. Because there are no upstream blade rows to distort the flow, the total pressure profile at the exit of the 1st vane is flat across most of the span as expected. However, the secondary flows near both endwalls distort the total pressure profile at $10 \%$ span near the hub and at $85 \%$ span near the tip.

The differences between the various predicted results are visible. The steady flow prediction matches the experimental data quite well in general with slight differences between $60 \%$ and $85 \%$ span. The location of both endwall passage vortices is also predicted well. 


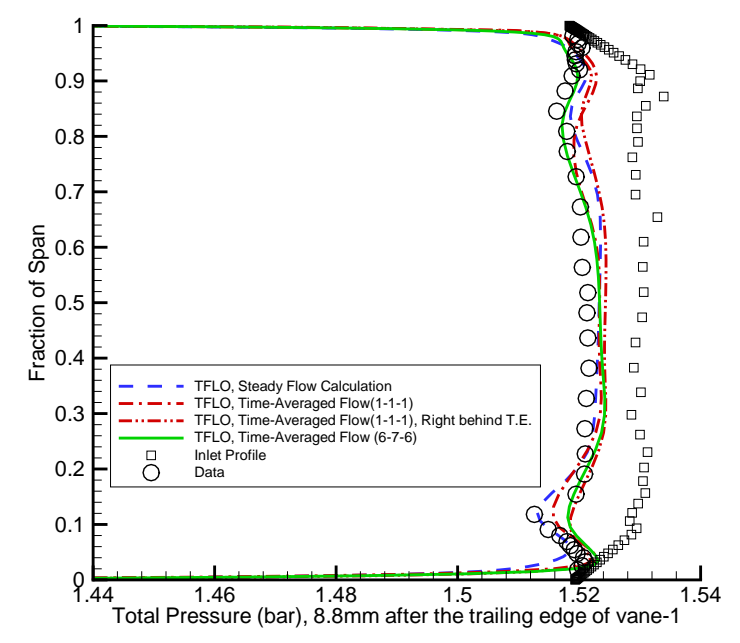

Fig. 3 Absolute total pressure comparison, 8.8 mm behind the trailing edge of vane-1

The time-averaged unsteady flow simulation for the 11-1 configuration predicts the tip passage vortex core location too far radially inwards. However, the total pressure profile from the same 1-1-1 simulation immediately downstream of the trailing edge agrees better with the experimental data. This indicates that the tip passage vortex shifts down $10 \%$ of the vane span and has a considerable amount of additional loss between $80 \%$ and $60 \%$ span. These effects are likely to be due to the increase in the blade size for the 1-1-1 configuration. The configuration 6-7-6 clearly improves the prediction of this vortex core and the total pressure profile as well.

There is a strong total pressure loss around 10\% span caused by the hub passage vortex. This vortex is captured by all the simulations although in different intensity. The total pressure peak near 3\% span is formed due to the fact that the hub passage vortex collects the low total pressure fluid in the inflow near the hub and pushes higher energy fluid downwards towards the endwall, and hence forms the edge of the endwall boundary layer. Unsteady calculations predict this peak very well, while the steady calculation tends to give a thicker endwall boundary layer which results in the predicted peak moving radially upwards.

Corresponding to the total pressure losses, the pitchwise flow angle downstream of the vane- 1 deviates from the mainstream value in the vicinity of the two passage vortices, as shown in Figure 4. Both unsteady calculations show improvement in predicting the flow angle over the steady prediction especially near $80 \%$ span where the tip passage vortex exists. Both the 6-7-6 and the 1-1-1 predictions are in fairly good agreement with the experimental data with the 6-7-6 simulation results being slightly better.

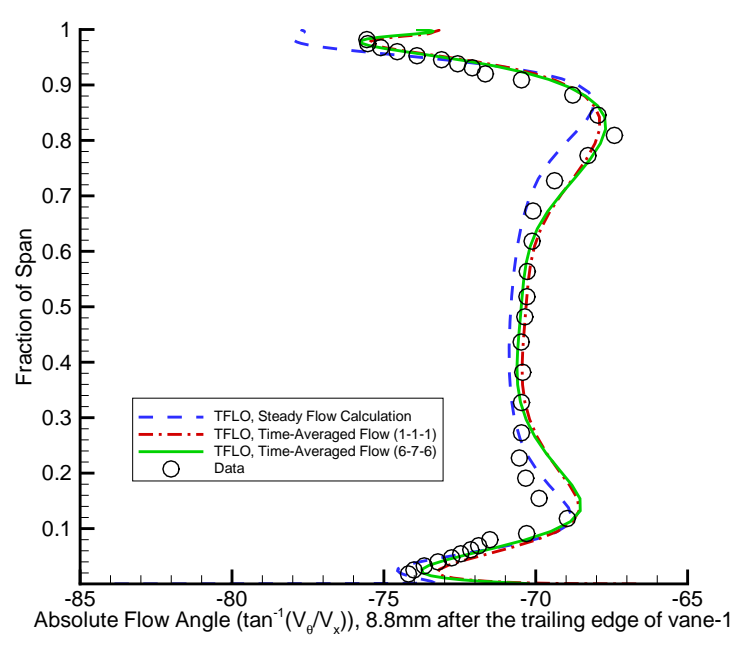

Fig. 4 Absolute flow angle comparison, $8.8 \mathrm{~mm}$ behind the trailing edge of vane-1

\section{Blade Exit}

The comparison of absolute total pressure at 8.8 $\mathrm{mm}$ downstream of the blade trailing edge is shown in Figure 5. None of the solutions are able to predict the absolute total pressure level at this station. This discrepancy may be due to experimental measurement problems. The spanwise trends in the predicted profile are fairly consistent and agree with the experimental data. The predictions do not capture the total pressure drop at $15 \%$ span and the peak at $8 \%$ span, however, where the rotor hub passage vortex exists.

Similar discrepancies as for the absolute total pressure exist between the predicted and experiment absolute total temperature levels as shown in Figure 6. Again, all of the predictions capture the spanwise trends of the experimental data. The unsteady simulation of the 1-1-1 configuration predicts the total temperature peak location (measured at $72 \%$ span) below the experimental data as a result of the blade geometry change. The unsteady flow calculation of the 6-7-6 configuration, however, shows slightly better agreement with the experimental temperature profile shape.

The profile of the pitch-wise absolute flow angle in Figure 7 shows the different secondary flow signatures downstream of the trailing edge more clearly. The change in flow angle between $22 \%$ to $32 \%$ span is caused by the upstream vane hub passage vortex. Unfortunately, all of the simulations merge this upstream vane hub passage vortex with that of the rotor hub. The change in flow angle between $60 \%$ to $75 \%$ span is caused by the rotor trailing edge vortex identified by Walraevens and Gallus. ${ }^{4}$ This change in flow angle is captured well by the unsteady calculation for the 6-76 configuration but the simulation underpredicts the strength. The simulation for the 1-1-1 configuration 


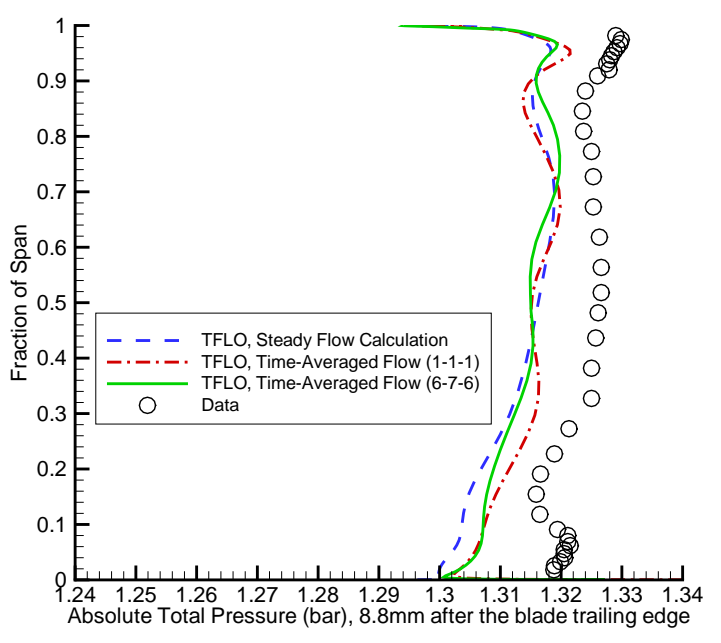

Fig. 5 Absolute total pressure comparison, 8.8 $\mathrm{mm}$ behind the trailing edge of the rotor

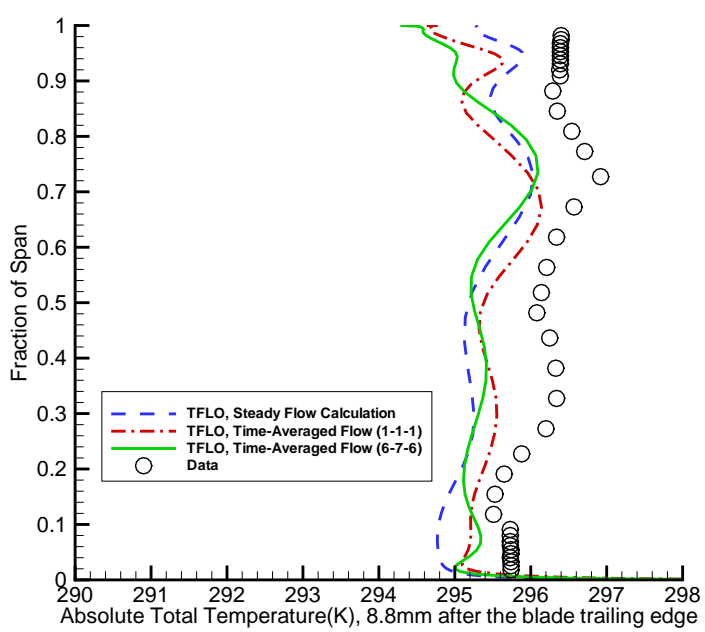

Fig. 6 Absolute total temperature comparison, 8.8 $\mathrm{mm}$ behind the trailing edge of the rotor

also captures this change in flow angle but the spanwise location is too far inward radially. The change in flow angle between $90 \%$ to $95 \%$ span is caused by the upstream vane tip passage vortex and the rotor tip vortex. Again, the 6-7-6 simulation captures this flow angle change in the correct location while the 11-1 simulation predicts the passage vortices at a lower span location.

\section{Vane-2 Exit}

The geometry of the second vane is identical to the first vane, but the second vane receives a much more distorted inflow resulting from the effect of the first vane and the rotor. Figure 8 shows comparable profiles to the ones behind the first vane. For comparison purposes, the total pressure profile at the inlet to

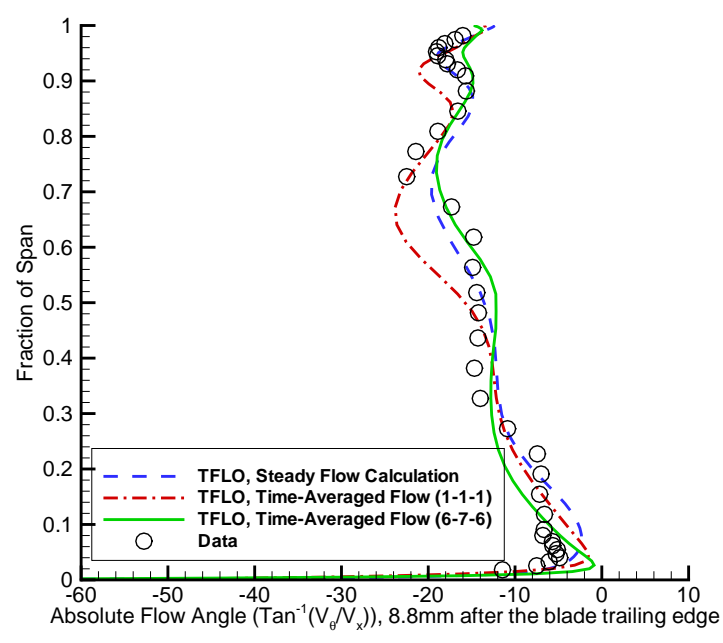

Fig. 7 Absolute flow angle comparison, $8.8 \mathrm{~mm}$ behind the trailing edge of the rotor

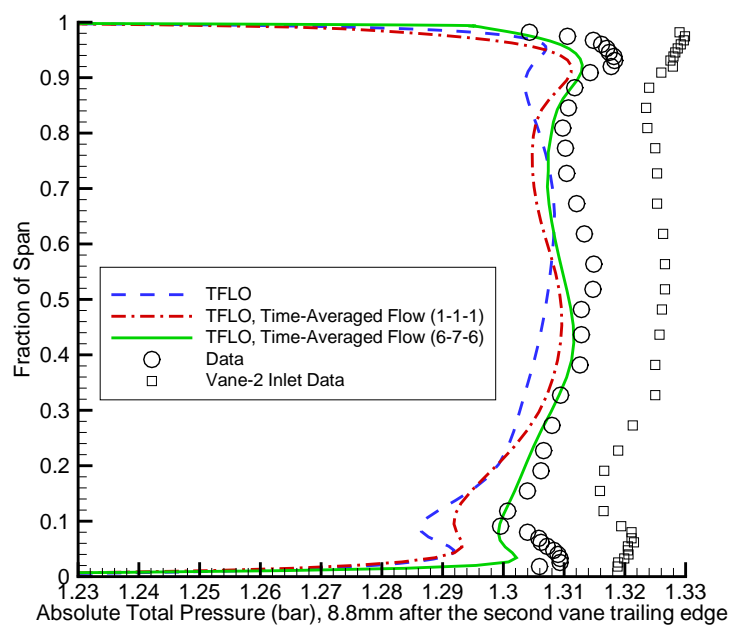

Fig. 8 Absolute total pressure comparison, 8.8 mm behind the trailing edge of vane-2

the second vane is included in this figure. The total pressure loss near the endwalls results from the accumulation of loss from all the blade rows. As before, the 6-7-6 configuration simulation improves the prediction near the endwalls (especially near the hub) over the 11-1 configuration and steady flow calculations due to the minimal geometry modifications and the modeling of the unsteady flow physics.

Examining the inlet and exit total pressure measurements for the second vane, Figure 8 shows that the peak loss at the inlet near the hub has been pushed towards the hub at the exit. The predictions re-capture the dip of the total pressure. Near the casing area, the radial movement of the loss core is just the opposite. The loss core has moved away from the endwall. The simulations predict this same characteristic. 


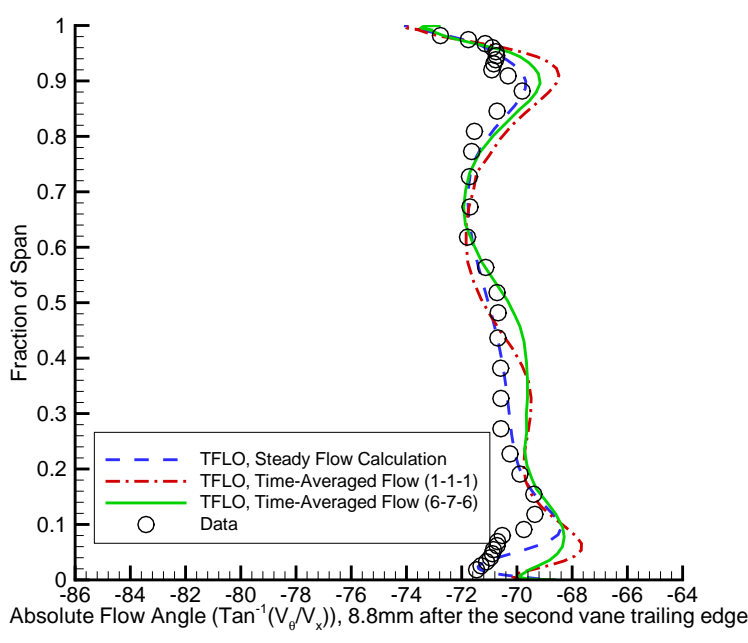

Fig. 9 Absolute flow angle comparison, $8.8 \mathrm{~mm}$ behind the trailing edge of vane-2

The pitch-wise absolute flow angle profiles at $8.8 \mathrm{~mm}$ behind the second vane trailing edge, however, are not that similar to the ones at the exit of the first vane as shown in Figure 9. The changes in flow angle near the endwalls are due to the vane- 2 hub and casing passage vortices. The change in flow angle between $50 \%$ to $60 \%$ span is the signature of the secondary flow in the rotor which is carried through the second vane. All of the simulations do a reasonably good job at predicting the flow angle distribution with the time-averaged 67-6 solution being slightly better than the other two.

\section{Time-Dependent Flow Characteristics}

In this section, comparisons between the predicted and experimental unsteady flow characteristics along with a discussion of the predicted unsteady modes are presented. The predicted total pressure losses are compared between the steady flow and two unsteady flow calculations.

\section{Instantaneous secondary flows at stator-2 outlet}

In this section, we will examine the unsteady secondary flow features at axial planes located at the exit of the second stator. The secondary flows play an important role in determining the loss mechanism. Generally, secondary flows decay more slowly than the wakes, hence have a stronger influence on the unsteadiness of the downstream blade rows.

The definition of secondary flow used in this paper follows that used by Walraevens and Gallus ${ }^{4}$ in order to perform similar comparisons with the experimental data: a secondary flow vector is defined as the difference between the local flow vector and an averaged flow vector. The latter is calculated using the total local velocity, the radially averaged yaw angle and the circumferentially averaged pitch angle. The two unsteady calculations are compared to the measurement at the closest time indices, as shown in Figure 10.

Close comparison of the predicted and experimental vectors show that the 6-7-6 simulation nearly always predicts the experimental features better than the 1-1-1 simulation and agrees very well with the experimental data. In fact, the 1-1-1 simulation fails to predict many of the unsteady flow vortices that can be observed in the experiment and 6-7-6 simulation. These unsteady vortices are responsible for the different losses in the flow compared to the predicted steady flow as well as changes in the radial migration of these losses. This comparison shows the importance of minimizing any geometry modifications when performing unsteady flow simulations in order to accurately predict the unsteady flow and loss transport characteristics.

\section{Instantaneous Mach number at stator-2 outlet}

The uniform flow at the inlet of the turbine is turned and distorted as it goes through the three blade rows. At the outlet of the last blade row, the instantaneous Mach number contours reflect all of the flow patterns. Comparisons are made between the measurement and the solution from the 6-7-6 configuration at 8 different time indices, as shown in Figure 11. The prediction shows many of the same features as the experimental data and once again shows the ability of the 6-7-6 simulation to accurately model the migration of the various losses in the flow.

\section{Prediction of the total pressure losses}

The total pressure loss is defined as

$$
\omega=\frac{P_{0}-P}{P_{0}},
$$

where $P_{0}$ is the circumferentially and radially massaveraged total pressure at inlet of a blade row, and $P$ is the total pressure of the point of interest. The overall total pressure loss is calculated using a circumferentially and radially mass-averaged total pressure at exit of a blade row as the value of $P$. These losses are listed in Table 1. Even though the losses of the steady flow calculation and the unsteady flow calculation of the 1-1-1 configuration are generally larger than the losses of the 6-7-6 configuration, they still remain on the same level.

The unsteady pattern of the loss distribution, however, shows great differences corresponding partly to the secondary flow features. Figure 12 shows the instantaneous contours of relative total pressure loss coefficient in the axial gap behind the rotor at $t=0.25 \mathrm{~T}$. Figure 13 shows the instantaneous contours of relative total pressure loss coefficient in the axial gap behind the second stator at $t=0.25 T$. The features in the loss contours at both locations are different between the 1-1-1 and the 6-7-6 simulations due to the different unsteady loss transport previously described. 


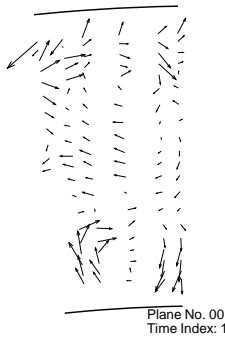

a) Experiment

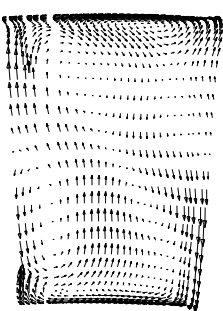

b) Config. 1-1-1

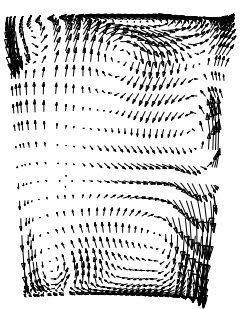

c) Config. 6-7-6

Time index: 1, Movement: $0 \%$ rotor pitch

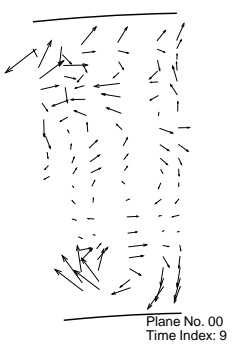

d) Experiment

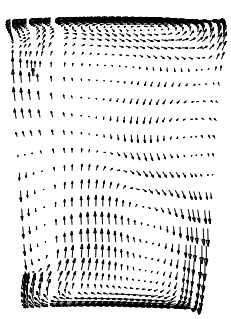

e) Config. 1-1-1

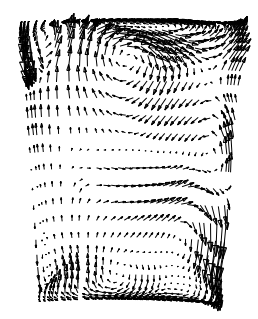

f) Config. 6-7-6 Time index: 9, Movement: $12.5 \%$ rotor pitch
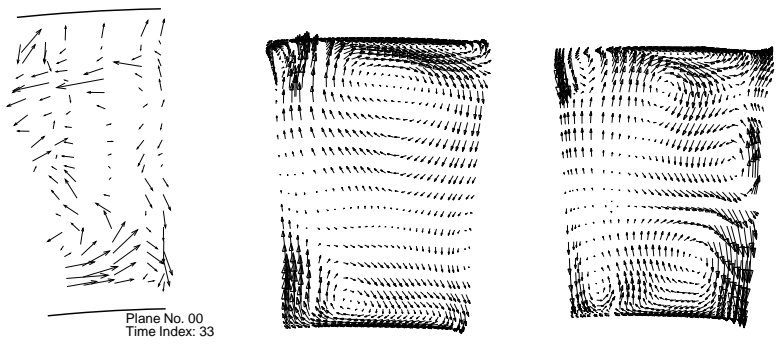

m) Experiment

n) Config. 1-1-1

o) Config. 6-7-6

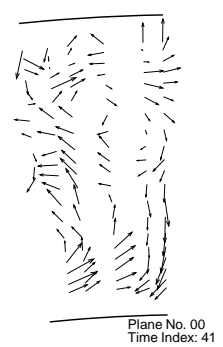

p) Experiment

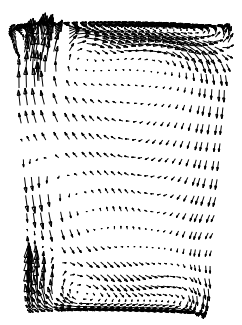

q) Config. 1-1-1

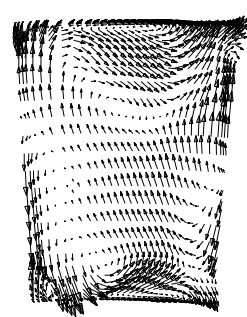

r) Config. 6-7-6

Time index: 41, Movement: $62.5 \%$ rotor pitch

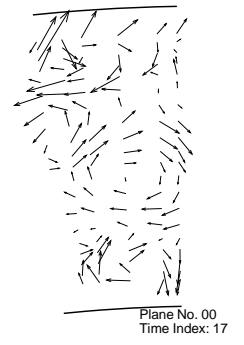

g) Experiment

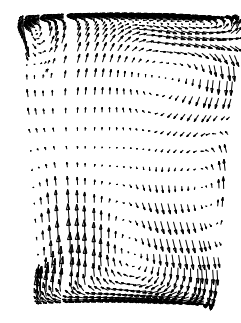

h) Config. 1-1-1

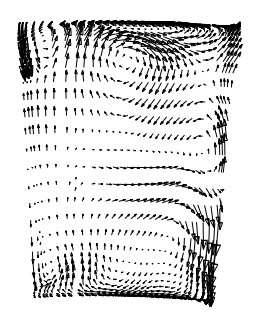

i) Config. 6-7-6

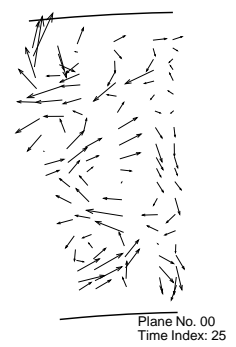

j) Experiment

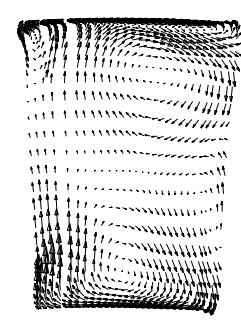

k) Config. 1-1-1

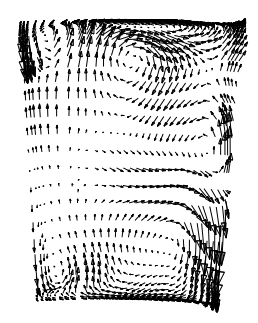

1) Config. 6-7-6 Time index: 25, Movement: $37.5 \%$ rotor pitch
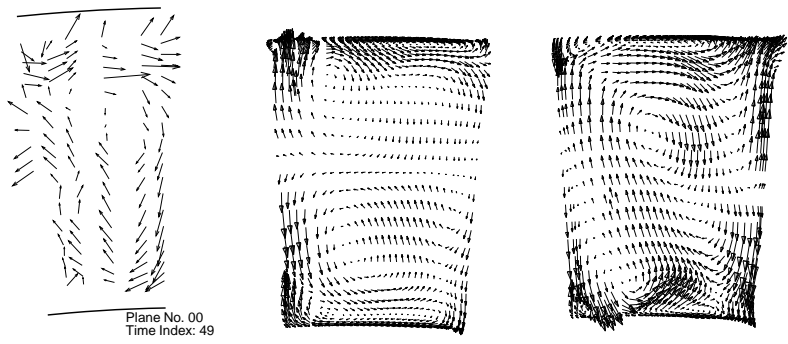

s) Experiment

t) Config. 1-1-1

u) Config. 6-7-6

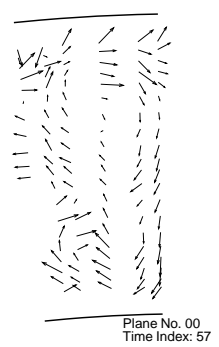

v) Experiment

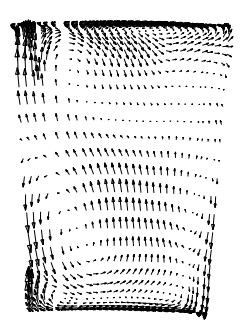

w) Config. 1-1-1

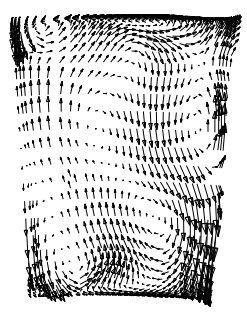

x) Config. 6-7-6

Fig. 10 Comparison of secondary flow at the outlet of vane-2 


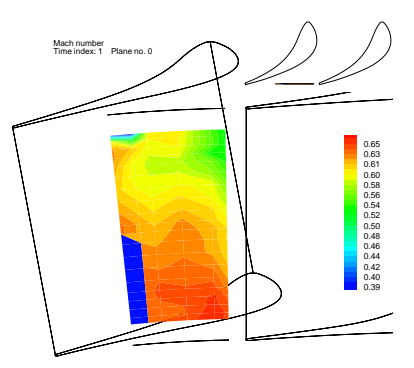

a) Experiment

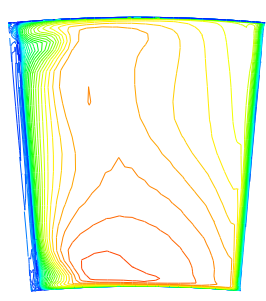

b) Config. 6-7-6

Time index: 1, Movement: $0 \%$ rotor pitch

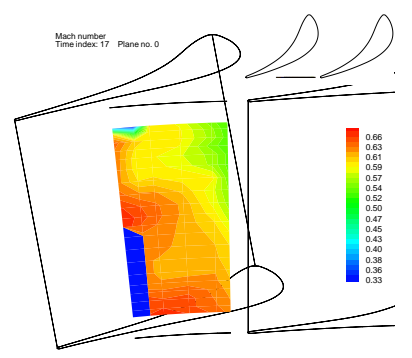

c) Experiment

Time index: 17, Movement: 25\% rotor pitch
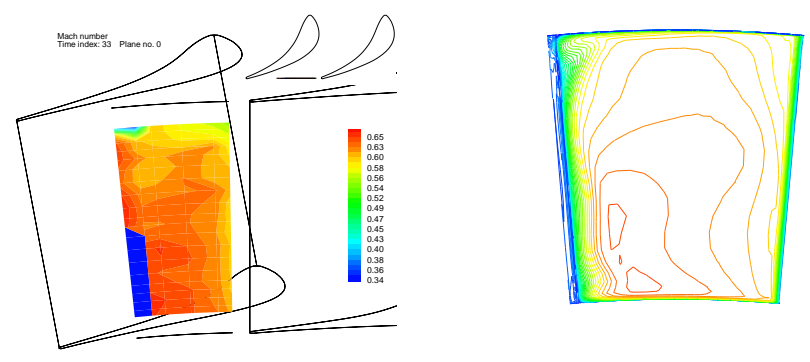

e) Experiment

f) Config. 6-7-6

Time index: 33, Movement: 50\% rotor pitch
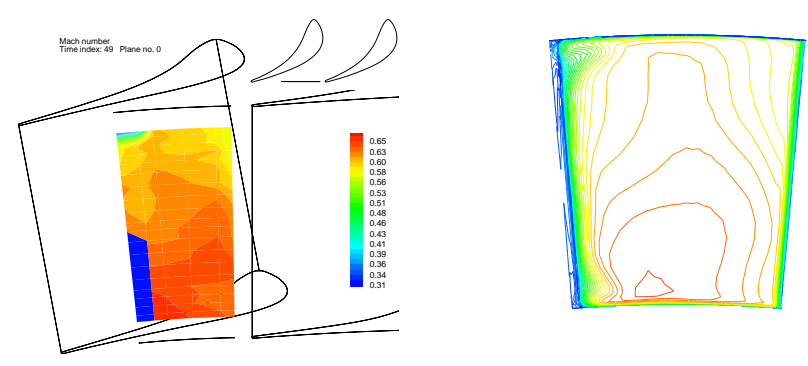

g) Experiment

h) Config. 6-7-6

Time index: 49, Movement: $75 \%$ rotor pitch

Fig. 11 Comparison of instantaneous Mach numbers at the outlet of vane-2

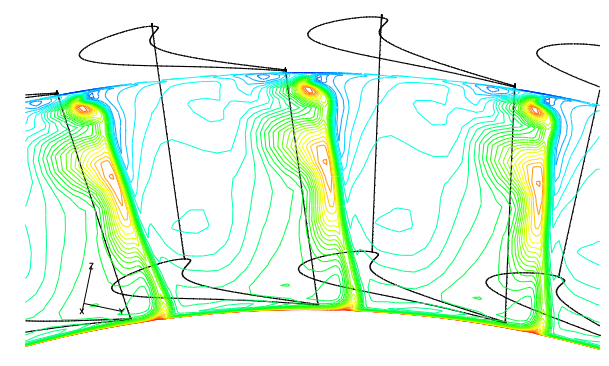

a) Configuration 1-1-1

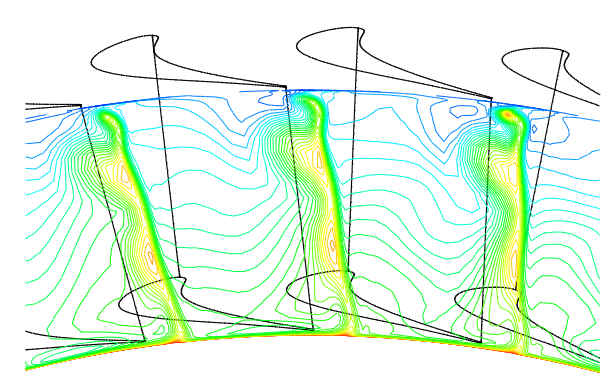

b) Configuration 6-7-6

Fig. 12 Instantaneous relative total pressure loss, downstream of the rotor, $t=0.25 T$

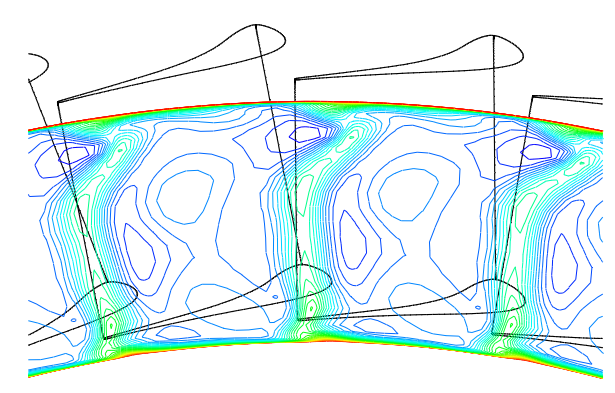

a) Configuration 1-1-1

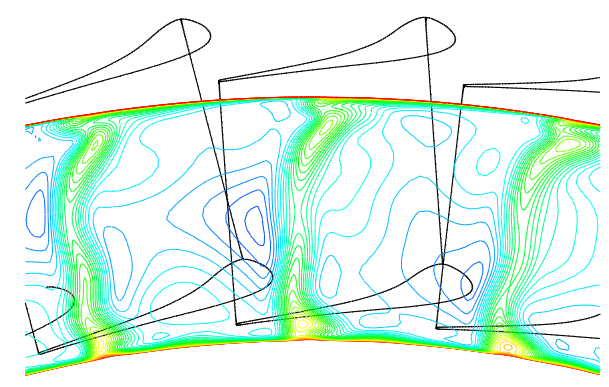

b) Configuration 6-7-6

Fig. 13 Instantaneous relative total pressure loss, downstream of the second stator, $t=0.25 T$ 


\begin{tabular}{||c|c|c|c||}
\hline \hline & Vane-1 & Blade & Vane-2 \\
\hline Steady flow & $0.654 \%$ & $0.725 \%$ & $0.763 \%$ \\
\hline Config. 1-1-1 & $0.670 \%$ & $0.633 \%$ & $0.962 \%$ \\
\hline Config. 6-7-6 & $0.453 \%$ & $0.481 \%$ & $0.956 \%$ \\
\hline \hline
\end{tabular}

Table 1 Predicted relative total pressure losses of each blade row

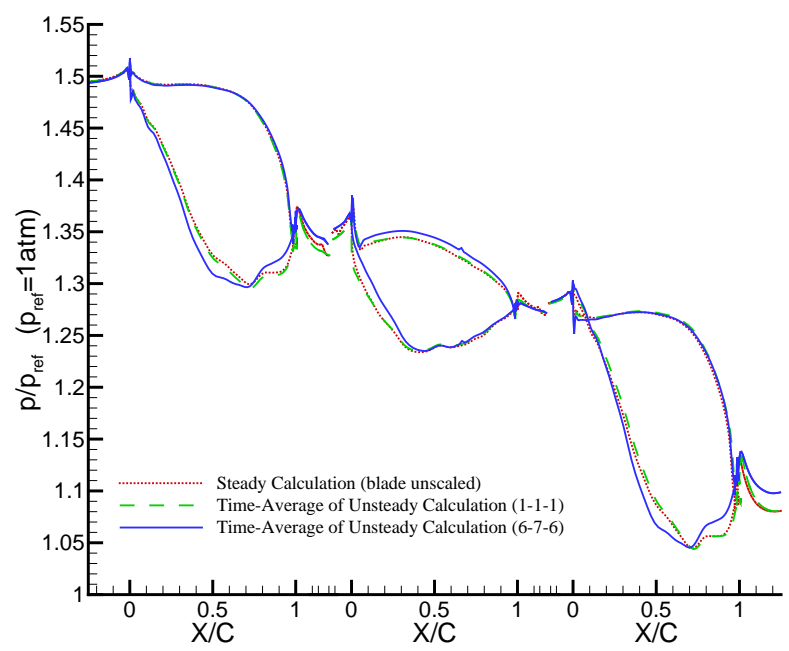

Fig. 14 Static pressure on blade surfaces at midspan

\section{Instantaneous Pressure on Blade Surfaces}

The surface pressure distributions are of great importance for blade design. The time-averaged pressure distribution is important in terms of determining the "steady" forces and moments on each blade. As previously discussed, the unsteady pressure forces on the blades are critical in determining the maximum (peak) stress levels and whether any of the unsteady modes can lead to resonance. Since the 6-7-6 simulation has been shown to more accurately predict the timeaveraged and unsteady flow fields, comparison with the 1-1-1 simulation can be used to further quantify the differences in the unsteady forces due to blade scaling.

\section{Time-averaged pressure and pressure envelopes}

Figure 14 shows the surface pressures at mid-span predicted by the steady flow and time-averaged unsteady calculations of the two configurations. The results from the 1-1-1 configuration are very similar to the pressure predicted by the steady-state calculation especially for the two stators. However, on the suction surfaces of the two stators, and on both surfaces of the rotor, the unsteady calculation of the 6-7-6 configuration shows different profiles in the vicinity of the lowest pressure point.

The unsteady pressure envelopes at mid-span are also shown in Figure 15. The rear part of the suction

\begin{tabular}{||c|c|c|c||}
\hline \hline & Vane-1 & Blade & Vane-2 \\
\hline Config. 1-1-1 & $0.29 \%$ & $1.48 \%$ & $0.77 \%$ \\
\hline Config. 6-7-6 & $0.31 \%$ & $1.54 \%$ & $0.79 \%$ \\
\hline \hline
\end{tabular}

Table 2 Predicted maximum amplitudes of pressure signals on blade surfaces, normalized by 1 atm

surface of the first vane has the largest pressure envelope for that blade row. The calculation of the 6-7-6 configuration shows a small but detectable difference compared with the result of the 1-1-1 configuration. The blade is affected throughout the whole surface due to the scaling difference of the two simulations. However, the pressure envelopes agree fairly well with each other. Interestingly, the 6-7-6 calculation shows a flatter profile on the blade suction surface than that of the 1-1-1 calculation. The envelope of the second vane shows a similar pattern to that of the first vane, but with a larger amplitude of pressure oscillations. Overall, the pressure envelopes of the two simulations are in fairly close agreement with each other. This would lead to the wrong conclusion, as will be discussed below, that the blade count scaling differences between the two simulations did not result in much of a change in the unsteady pressure; as will be shown below, however, the frequency content and the unsteady pressure amplitudes are different in the two simulations once again leading to the conclusion that blade scaling should be kept to a minimum in order to accurately predict unsteady pressure modes and resonance stress levels.

\section{Unsteady pressure characteristics}

The unsteady pressure signals on the blade surfaces are post-processed using Fast Fourier Transforms (FFT) to obtain information in the frequency domain. The amplitudes are normalized by the maximum value of the whole spectrum of each blade row. Each blade is unfolded along the trailing edge line for plotting purposes. Comparisons are made between the 1-1-1 and 6-7-6 simulations. The overall maximum amplitudes of the pressure waves are predicted similarly between the 1-1-1 and 6-7-6 simulations, as shown in Table 2. As will be shown below, however, the local amplitudes of the unsteady pressure waves can vary significantly between the two simulations.

\section{Vane-1}

Figures 16 and 17 show the normalized amplitudes of the pressure oscillations on the suction and pressure surfaces of the first vane at the one blade passing frequency $(1 \mathrm{BPF})$ and at twice the blade passing frequency $(2 \mathrm{BPF})$, respectively. The $1 \mathrm{BPF}$ and $2 \mathrm{BPF}$ contour shapes are similar between the 1-1-1 and 6-76 simulations but, as will be further shown below, local amplitudes for the 1-1-1 simulation can be quite different from those for the 6-7-6 simulation at the same 


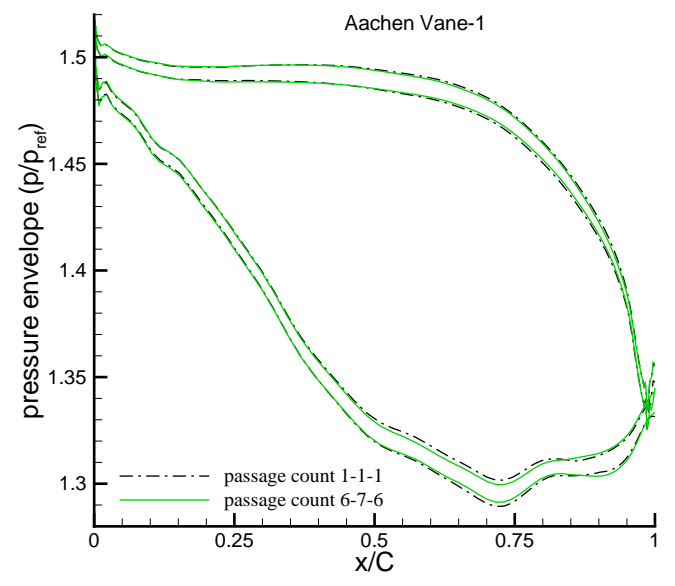

a) Vane-1

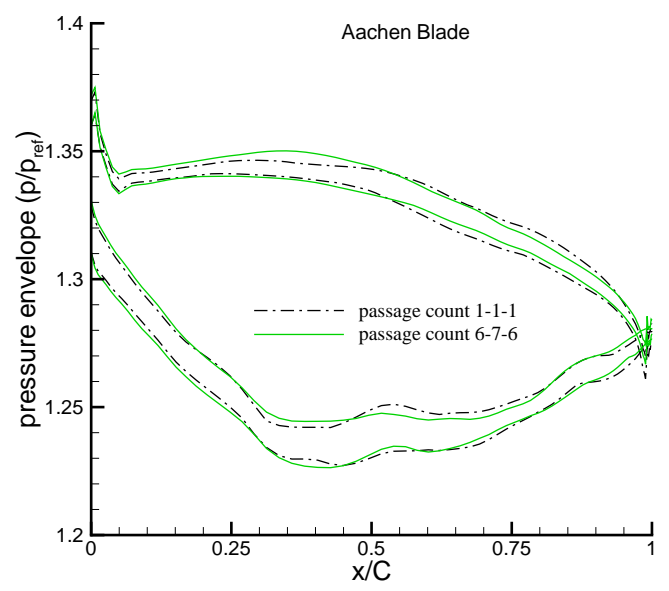

b) Blade

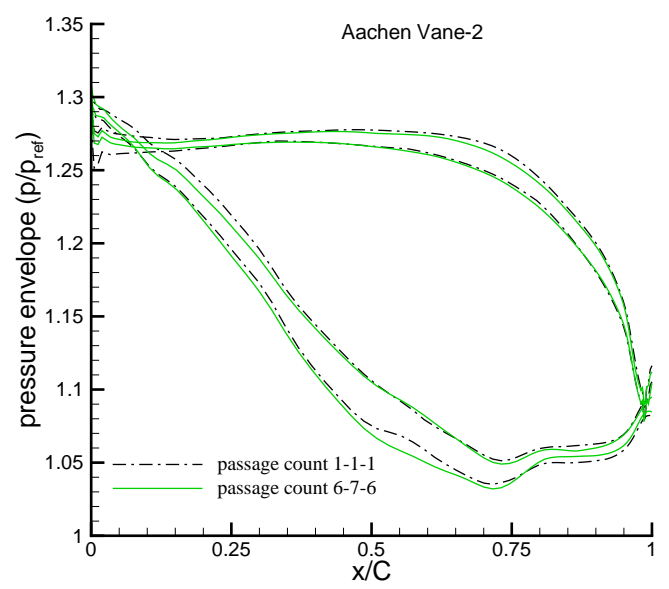

c) Vane-2

Fig. 15 Comparison of unsteady pressure envelopes at mid-span

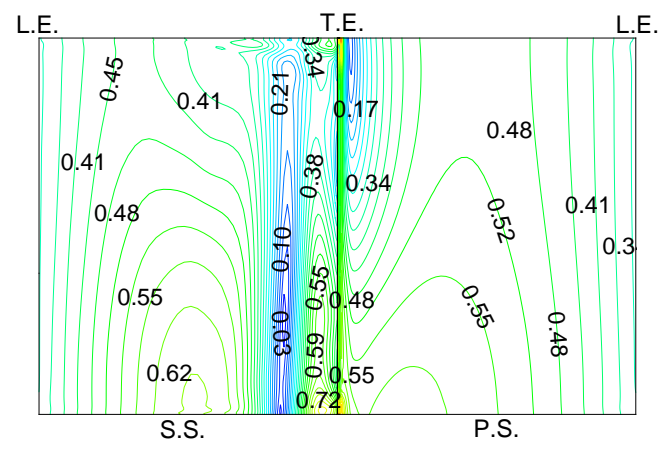

a) Config. $1-1-1$ at $1 \mathrm{BPF}$

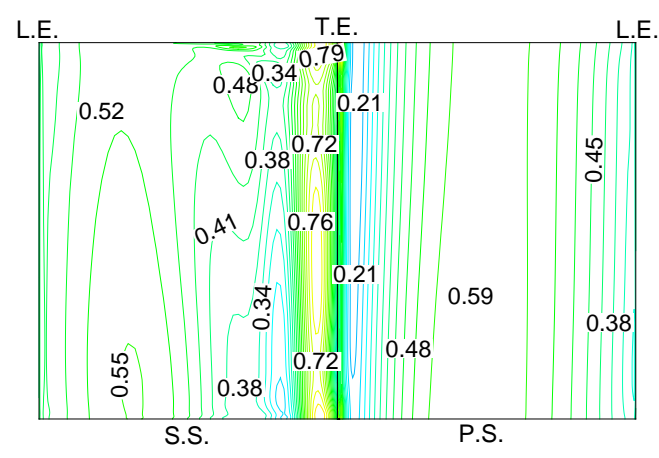

b) Config. 6-7-6 at $1 \mathrm{BPF}$

Fig. 16 Comparison of normalized pressure amplitudes on vane-1 surfaces at $1 \mathrm{BPF}$

location.

The simulation of the 1-1-1 configuration can only pick up the frequency content of an integer multiple of the BPF because one blade passage was used for every blade row. The results from the 6-7-6 configuration not only show somewhat different patterns than those for 1-1-1 configuration, but the 6-7-6 calculation also admits much fuller frequency content in between the blade passing harmonics. Figure 18 shows the amplitude of the frequency spectrum at mid-span near the trailing edge of the vane- 1 suction side of the 6-7-6 configuration. The 6-7-6 simulation resolves pressure oscillations in between the 1st and 2nd harmonics. It also resolves lower harmonics (frequencies less than $1 \mathrm{BPF})$. As indicated in the figure, this capturing of non-integer blade passing frequencies is not related to the trailing edge vortex shedding. It represents the longer period (more than one passage) pressure waves reflecting between the blade rows.

\section{Blade}

Figures 19 and 20 show the normalized amplitude of the pressure oscillation on the blade suction and pressure surfaces at $1 \mathrm{BPF}$ and $2 \mathrm{BPF}$, respectively. The largest differences in the frequency amplitudes are in the end-wall regions, especially on the suction surface.

The low harmonics predicted by the 6-7-6 simulation are observed to have different magnitudes at different 


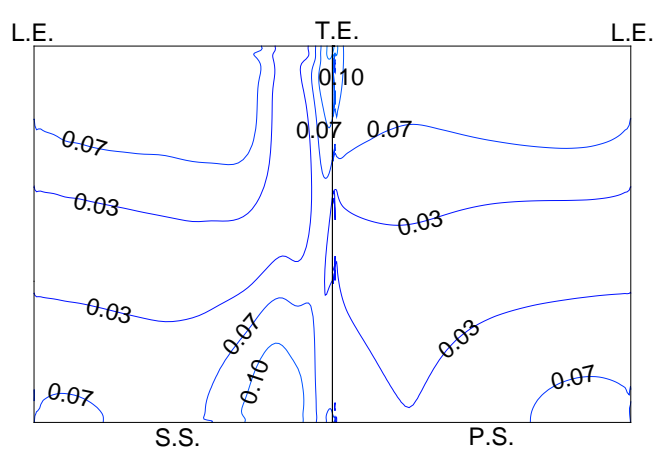

a) Configuration 1-1-1

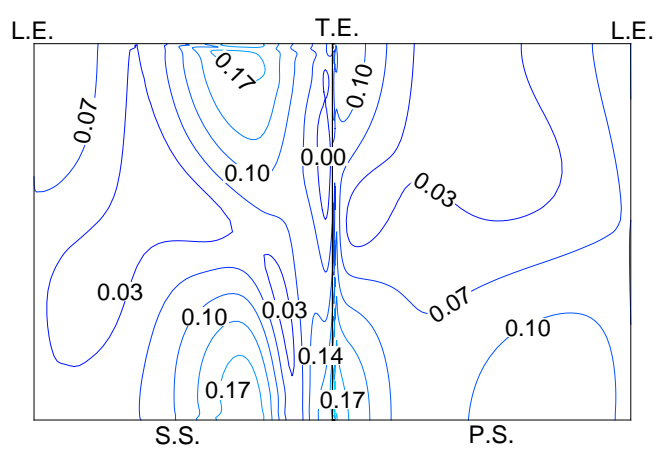

b) Configuration 6-7-6

Fig. 17 Comparison of normalized pressure amplitudes on vane-1 surfaces at $2 \mathrm{BPF}$

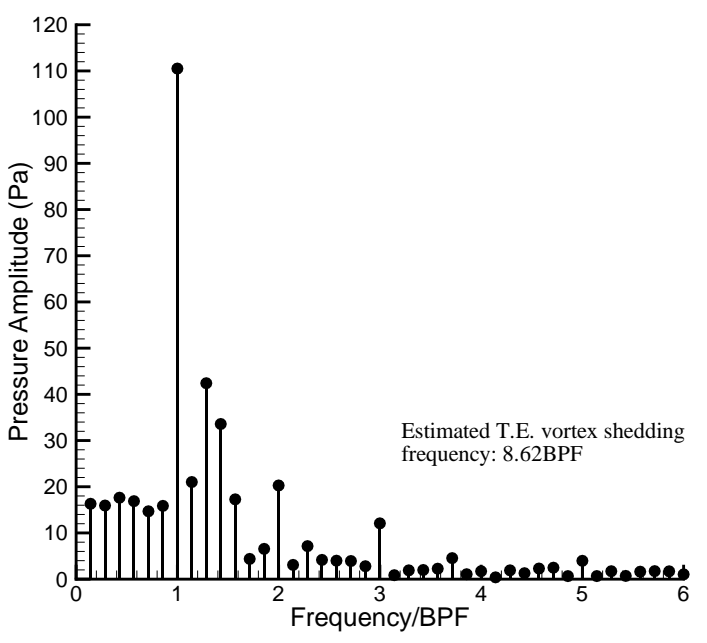

Fig. 18 Pressure harmonics of a point at mid-span, near T.E. of vane-1 suction surface

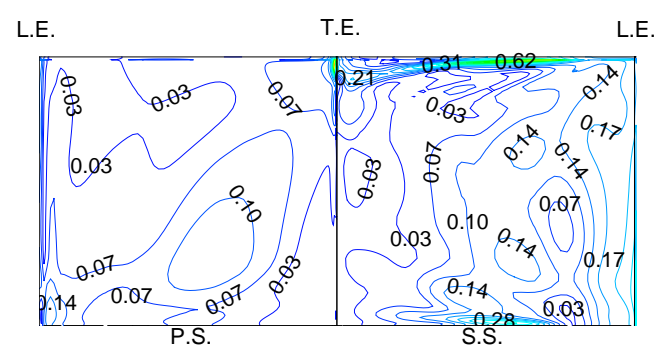

a) Configuration 1-1-1

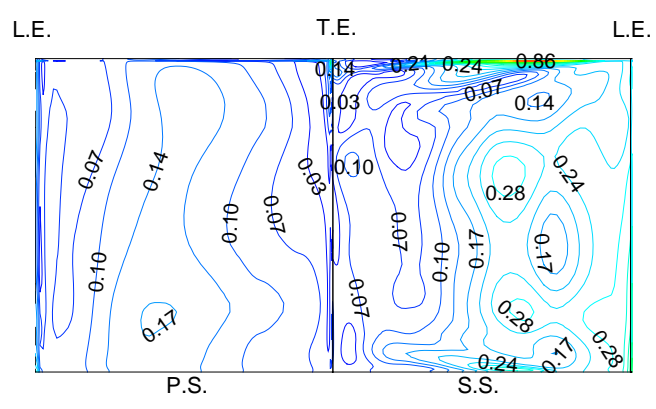

b) Configuration 6-7-6

Fig. 19 Comparison of normalized pressure amplitudes on rotor surfaces at $1 \mathrm{BPF}$

places on the blade surface. Figure 21 shows the FFT results of the frequency spectrum at a point near the trailing edge at mid-span on the pressure surface of the blade for both the 6-7-6 and 1-1-1 simulations. This figure shows not only that the 6-7-6 spectrum has much more frequency content but also that the amplitudes of the fundamental frequencies are quite different from those predicted in the 1-1-1 simulation. The first harmonic of the 1-1-1 simulation is twice that of the 6-7-6 but the second, third, and fourth harmonics are quite small relative to those of the 6-7-6 simulation. Once again, this shows that the local unsteady pressure amplitudes are quite dependent on the blade count scaling strategy.

Vane-2

Figures 22 and 23 show the normalized amplitude of the pressure oscillation on the suction and pressure surfaces of the second vane at $1 \mathrm{BPF}$ and $2 \mathrm{BPF}$, respectively. Once again, the $1 \mathrm{BPF}$ and $2 \mathrm{BPF}$ contours are similar in shape between the 1-1-1 and 6-7-6 simulations but local magnitudes can differ significantly.

The low harmonics (those below 1BPF) get even stronger in the third blade row. Figure 24 shows the FFT of the frequency spectrum at a point near the trailing edge at mid-span on pressure surface of the second vane. This figure shows that the low harmonics can be stronger than the $2 \mathrm{BPF}$ and that there are modes between the $1 \mathrm{BPF}$ and $2 \mathrm{BPF}$ that are even greater in magnitude. 


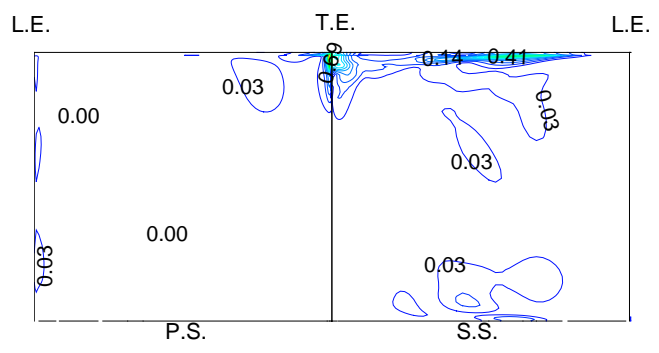

a) Configuration 1-1-1

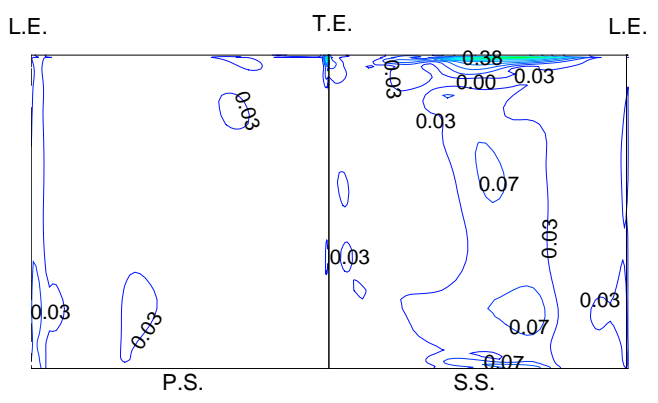

b) Configuration 6-7-6

Fig. 20 Comparison of normalized pressure amplitudes on rotor surfaces at $2 \mathrm{BPF}$

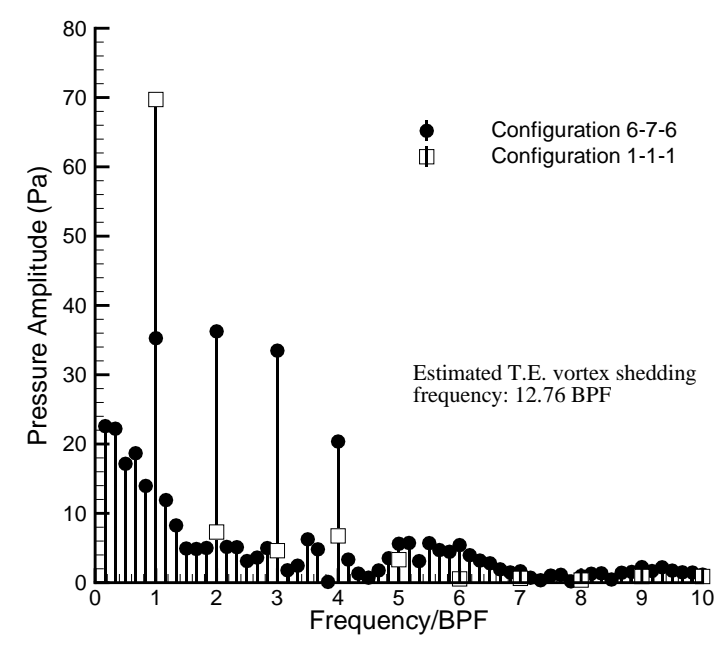

Fig. 21 Pressure harmonics of a point at mid-span, near T.E. of rotor pressure surface

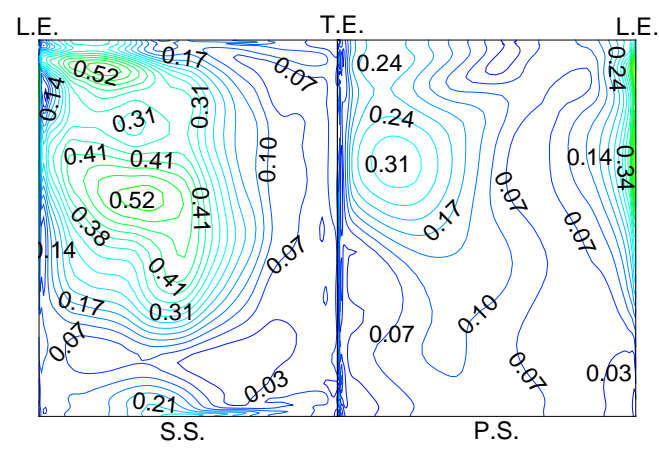

a) Configuration 1-1-1

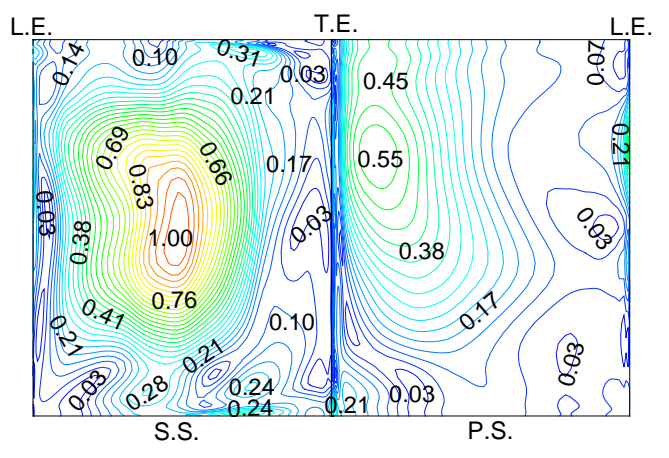

b) Configuration 6-7-6

Fig. 22 Comparison of normalized pressure amplitudes on vane-2 surfaces at $1 \mathrm{BPF}$

\section{Unsteady pressure in the rotor blade passage}

The blade's pressure field is affected by the upstream and downstream stators due to interactions with the potential fields of both blade rows and the reflections that result. In order to investigate the unsteady pressure waves inside the blade passage at a given spanwise (radial) location, the unsteady pressure signals on the circumferential plane at mid-span are decomposed in the frequency domain using FFTs and then re-constructed by using only the frequency content at $1 \mathrm{BPF}$ and $2 \mathrm{BPF}$ (relative to the blade row), respectively. This reconstruction at various time slices over the global period allows one to view the propagation of the $1 \mathrm{BPF}$ and $2 \mathrm{BPF}$ modes through the blade passage. The contour maps in 7 time slices over the global period are shown in Figure 25. The waves and their propagation are caused by upstream vane-1 passing wakes and the influence of the downstream stator leading edge potential field as well as reflections from the upstream and downstream vanes. It can be seen in Figure 25 that some of the waves propagate upstream and some propagate downstream.

\section{Conclusions}

An investigation in which two unsteady numerical simulations of a 1-1/2 stage axial subsonic flow turbine 


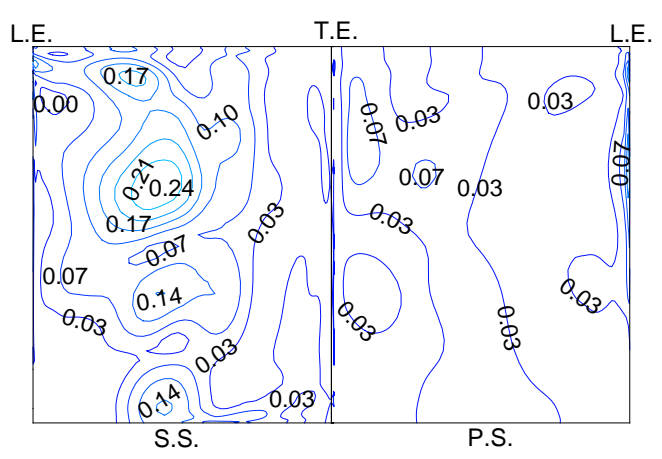

a) Configuration 1-1-1

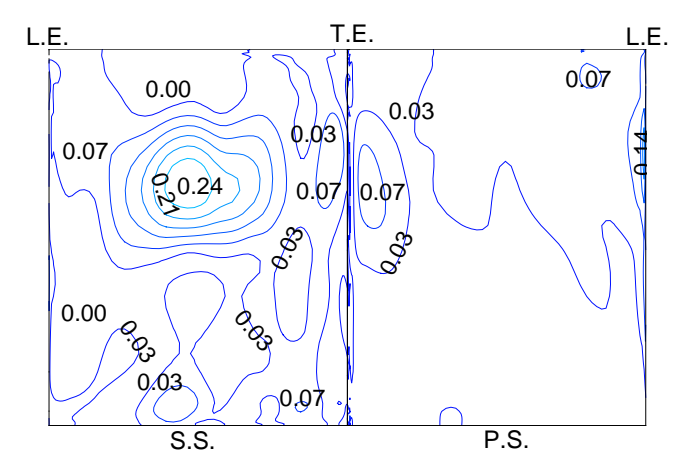

b) Configuration 6-7-6

Fig. 23 Comparison of normalized pressure amplitudes on vane-2 surfaces at $2 \mathrm{BPF}$

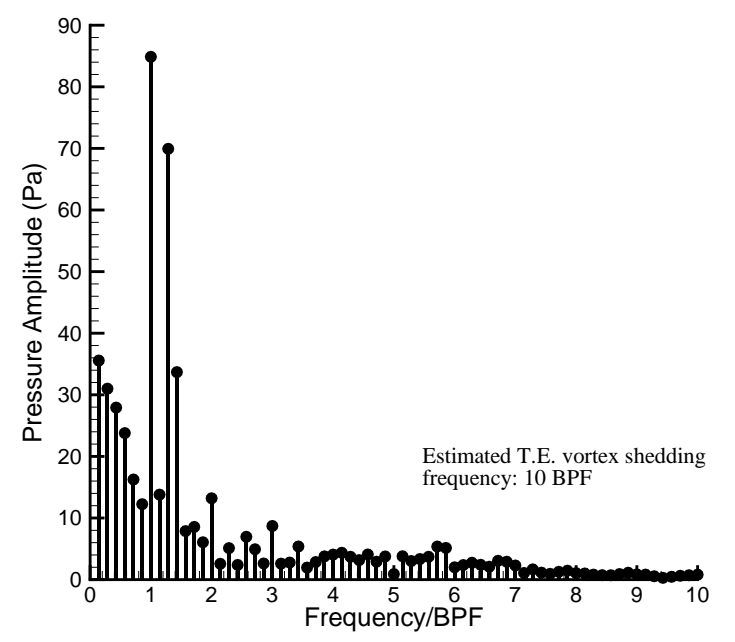

Fig. 24 Pressure harmonics of a point at mid-span, near T.E. on vane-2 pressure surface
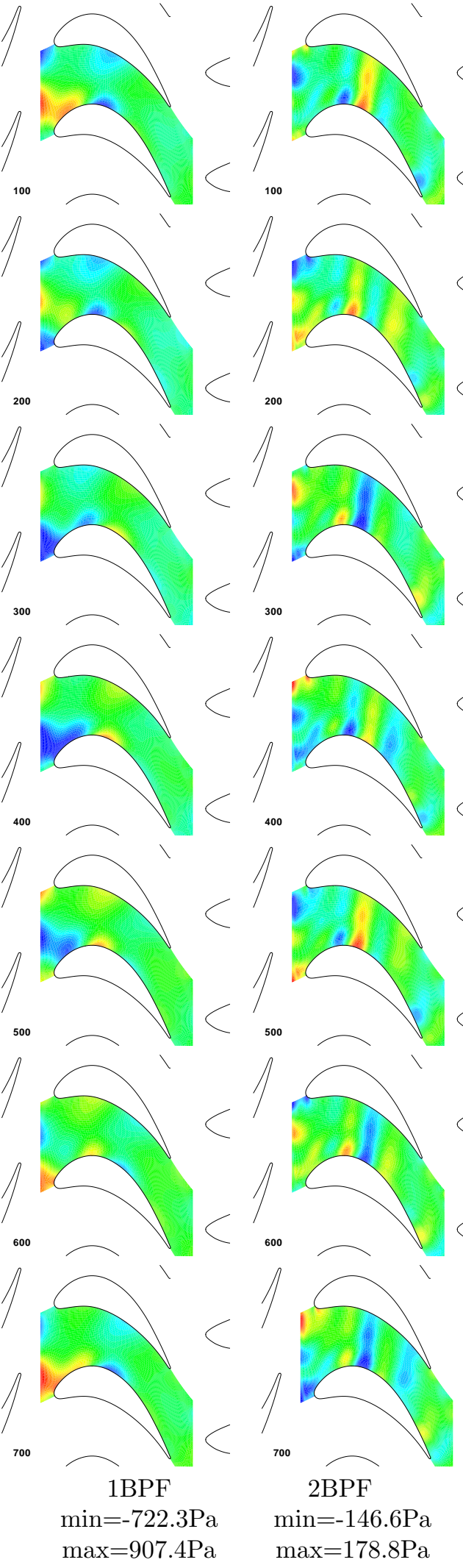

Fig. 25 Instantaneous pressure inside the rotor passage at mid-span. The pressures are reconstructed at $1 \mathrm{BPF}$ and $2 \mathrm{BPF}$ respectively by removing non-related frequencies. 
using 1-1-1 and 6-7-6 blade counts are compared with each other as well as with time-averaged and unsteady experimental data has been presented. The results from this investigation show that:

- the time-averaged pressure and unsteady pressure envelopes are not significantly affected by the blade count scaling strategy providing that the blade scaling is not excessive (i.e. $<14 \%$ for this case).

- the distribution as well as the integrated timeaveraged relative total pressure loss is significantly affected by the blade count scaling due to the unsteady transport of loss (entropy).

- harmonics of frequencies other than the blade passing frequency can be significant in these multi-stage flows and can only be predicted accurately if the blade counts in each blade row are modeled as closely as possible.

- it is important to minimize the airfoil scaling necessary to achieve a common circumferential period in order to accurately predict the unsteady pressure wave amplitudes that are required for accurate prediction of peak (resonance) stress levels of the blades

The use of large-scale, massively parallel computer systems is making it possible to simulate true blade counts over increasing numbers of blade rows in a turbomachine. New Reynolds-Averaged Navier-Stokes solution procedures, such as TFLO, are continuing to be developed to take advantage of these computer systems, validated, and demonstrated for large-scale turbomachinery applications.

\section{Acknowledgement}

The authors would like to thank the U.S. Department of Energy (DoE) for its generous support under the ASCI program. The authors would especially like to recognize the support of the staff and the Lawrence Livermore National Laboratory. We would also like to thank the managers at United Technologies Research Center and Pratt \& Whitney for their support. The ERCOFTAC (European Research Community On Flow, Turbulence And Combustion) is also gratefully acknowledged for providing the experimental data package for the Aachen case.

\section{References}

${ }^{1}$ Yao, J., Jameson, A., Alonso, J. J., and Liu, F., "Development and Validation of a Massively Parallel Flow Solver for Turbomachinery Flows," AIAA Paper 00-0882, Reno, NV, January 2000 .

${ }^{2}$ Jameson, A., "Time Dependent Calculations Using Multigrid, with Applications to Unsteady Flows Past Airfoils and Wings," AIAA Paper 91-1596, AIAA 10th Computational Fluid Dynamics Conference, Honolulu, HI, June 1991.
${ }^{3}$ Wilcox, D. C., Turbulence Modeling for CFD, DCW Industries, Inc., La Cañada, CA, 1998.

${ }^{4}$ Walraevens, R. E. and Gallus, H. E., "Three-Dimensional Structure of Unsteady Flow Downstream the Rotor in a 1-1/2 Stage Turbine," Unsteady Aerodynamics and Aeroelasticity of Turbomachines, 1995, pp. 481-498.

${ }^{5}$ Emunds, R., Jennions, I. K., Bohn, D., and Gier, J., "The Computation of Adjacent Blade-Row Effects in a 1.5 Stage Axial Flow Turbine," ASME Paper 97-GT-81, International Gas Turbine and Aeroengine Congress, 1997.

${ }^{6}$ Walraevens, R. E., Gallus, H. E., Jung, A. R., Mayer, J. F., and Stetter, H., "Experimental and Computational Study of the Unsteady Flow in a 1.5 Stage Axial Turbine with Emphasis on the Secondary Flow in the Second Stator," ASME Paper 98GT-254, International Gas Turbine and Aeroengine Congress, 1998.

${ }^{7}$ Volmar, T. W., Brouillet, B., Gallus, H. E., and Benetschik, H., "Time Accurate 3D Navier-Stokes Analysis of a 1-1/2 Stage Axial Flow Turbine," AIAA Paper 98-3247, 34th AIAA/ASME/SAE/ASEE Joint Propulsion Conference, 1998.

${ }^{8}$ Erdos, J. L., Alzner, E., and McNally, W., "Numerical Solutions of Periodic Transonic Flow Through a Fan Stage," AIAA Journal, Vol. 15, No. 1, 1977, pp. 1559-1568.

${ }^{9}$ Giles, M. B., "UNSFLO: A Numerical Method for Unsteady Inviscid Flow in Turbomachinery," MIT Gas Turbine Laboratory 195, 1988.

${ }^{10}$ Clark, J. P., Stetson, G. M., Magge, S. S., Ni, R. H., Jr., C. W. H., and Dunn, M. G., "The Effect of Airfoil Scaling on the Predicted Unsteady Loading on the Blade of a 1 and $1 / 2$ Stage Transonic Turbine and a Comparison with Experimental Results," ASME Paper 2000-GT-0446, ASME Turbo Expo 2000, Munich, Germany, May 2000.

${ }^{11}$ Yao, J., Jameson, A., Alonso, J. J., and Liu, F., "Development and Validation of a Massively Parallel Flow Solver for Turbomachinery Flows," AIAA Journal of Propulsion and Power, 2001, to be published. 